\title{
Suppression of Aggressive Strains of 'Candidatus Phytoplasma mali' by Mild Strains in Catharanthus roseus and Nicotiana occidentalis and Indication of Similar Action in Apple Trees
}

\author{
Bernd Schneider, Sandor Sule, Wilhelm Jelkmann, and Erich Seemüller
}

First, third, and forth authors: Julius Kuehn Institute, Federal Research Centre for Cultivated Plants, Institute for Plant Protection in Fruit Crops and Viticulture, D-69221 Dossenheim, Germany; and second author: Plant Protection Institute of the Hungarian Academy of Science, H-1525, Budapest.

Accepted for publication 21 October 2013.

\begin{abstract}
Schneider, B., Sule, S., Jelkmann, W., and Seemüller, E. 2014. Suppression of aggressive strains of 'Candidatus Phytoplasma mali' by mild strains in Catharanthus roseus and Nicotiana occidentalis and indication of similar action in apple trees. Phytopathology 104:453-461.

To study antagonistic interactions of 'Candidatus Phytoplasma mali' strains, graft inoculation of Catharanthus roseus and Nicotiana occidentalis was performed with mild strains 1/93Vin and 1/93Tab as suppressors and three aggressive strains as challengers. Inoculation of the suppressors was carried out in either the cross-protection modus prior to grafting of the challengers or by co-inoculating suppressors and challengers. Monitoring using multiplex real-time polymerase chain reaction assays revealed that, in long-term cross-protection trials with $C$. roseus, suppressor $1 / 93$ Vin was present in all root and randomly collected stem samples over

the entire observation period. In contrast, the challengers were never detected in such stem samples and rarely in the roots. Following simultaneous inoculation, the suppressor successively colonized all stem and root regions whereas detection of challenger AT steadily decreased. However, this strain remained detectable in up to 13 and $27 \%$ of stem and root samples, respectively. The cross-protection trials with $N$. occidentalis yielded results similar to that of the cross-protection experiments with C. roseus. Comparison of the symptomatology of infected apple trees with the presence of putatively suppressive strains indicated that suppression of severe strains also occurs in apple. Phylogenetic analysis using a variable fragment of AAA+ ATPase gene AP460 of ' $\mathrm{Ca}$. P. mali' revealed that suppressors $1 / 93 \mathrm{Vin}$ and 1/93Tab, together with several other mild strains maintained in apple, cluster distantly from obviously nonsuppressive strains that were predominantly highly virulent.
\end{abstract}

Apple proliferation (AP), caused by the wall-less bacterium 'Candidatus Phytoplasma mali', is one of the most important phytoplasmal diseases in Europe. This psyllid-transmitted disease induces a range of symptoms that are either specific (such as witches'-brooms, enlarged stipules, and undersized fruit with low eating quality) or nonspecific (such as foliar reddening or yellowing and reduced vigor). As previously reported, symptom expression depends on the virulence of the infecting strains. After 12 years of observation, 24 randomly collected ' $\mathrm{Ca}$. P. mali' accessions were classified as being avirulent or mildly virulent, moderately virulent, or strongly virulent. These groups occurred in approximately the same frequency. Whereas strongly virulent accessions caused severe stunting and decline of the trees, vigor and performance of trees infected by the first group were virtually indistinguishable from healthy controls and showed only occasional mild symptoms that did not markedly affect vigor or size and quality of the crop (23).

However, symptom expression is often subject to fluctuation. In particular, the formation of the most characteristic witches'broom symptoms is often typical for newly diseased trees and can be observed in the first few years of disease. Then, trees may recover and show no or only mild symptoms for shorter or longer

Corresponding author: E. Seemüller; E-mail address: erich.seemueller@jki.bund.de

* The $\boldsymbol{e}$-Xtra logo stands for "electronic extra" and indicates that Figure 1 appears in color online.

http://dx.doi.org/10.1094/PHYTO-08-13-0230-R

This article is in the public domain and not copyrightable. It may be freely reprinted with customary crediting of the source. The American Phytopathological Society, 2014 periods, after which severe symptoms may reappear $(1,21,22)$. From recent work, there is evidence that such fluctuations can be attributed to multiple infections of apple trees by distinct strains of ' $\mathrm{Ca}$. P. mali'. Such infections are common and are usually composed of strains that greatly differ in virulence. Obviously, due to antagonistic interactions between mild and severe strains, shifts in the population may occur that result in increased or, more often, reduced virulence of the infecting phytoplasmas $(20,22)$.

An example for such variations is our multiple-strain accession $1 / 93$. Trees infected by this source were largely symptomless for many years. Following transmission to periwinkle (Catharanthus roseus) and Nicotiana occidentalis, two distinctly different strains, referred to as $1 / 93 \mathrm{Vin}$ and $1 / 93 \mathrm{Tab}$, respectively, were identified by single-strand conformation polymorphism (SSCP) and sequence analysis of a variable fragment of $h f l B$ protease gene AP464. The same strains were identified in the largely symptomless donor tree of accession 1/93, jointly or separately. Each of the two strains identified in apple and the experimental hosts showed the distinct SSCP profile and clustered with other mild strains in phylogenetic analysis $(20,24)$. However, following graft transmission of accession 1/93 from a field-grown tree to clonal rootstocks and growth of the recipient trees in an unheated greenhouse, one of five trees developed witches'-broom symptoms. Using SSCP and sequence typing, a third strain was identified in the diseased tree that clustered with severe strains of other accessions in phylogenetic analysis. Further investigations revealed that this strain also occurred in low numbers in the roots of the symptomless donor tree, together with the two predominant mild strains. In addition, two other rarely detected genotypes were identified in 1/93-infected apple trees that clustered with severe strains of other accessions $(20,21)$. 
The HflB protease encoded by gene AP464 belongs to the large family of ATPases associated with various cellular activities $(\mathrm{AAA}+)$ proteins that include, in addition to characteristic ATPases, proteases having the structurally conserved ATPase domain together with the protease module $(7,26)$. The distinct phylogenetic clustering of mild and severe strains of ' $\mathrm{Ca}$. P. mali' based on the variable fragment of the HflB protease gene AP464 was also observed in genes encoding other AAA+ proteins of this phytoplasma. Analysis of the deduced protein sequences of the nine distinct AAA+ proteins present in ' $\mathrm{Ca}$. P. mali' revealed that the sequences from mild and severe strains of three of the four ATPases and three of the five HflB proteases cluster distantly, according to their virulence (24).

In this article, we report on further indication of antagonistic interactions between AP phytoplasma strains, mainly on the suppressive action of the largely avirulent periwinkle- and tobaccomaintained strains 1/93 Vin and 1/93Tab on aggressive strains available in these experimental hosts. The occurrence of suppressive actions of AP phytoplasma strains in infected apple trees was inferred by linking SSCP and nucleotide sequence data of the AAA+ ATPase gene AP460 with the disease history of the trees and the symptoms shown at sampling. The ATPase AP460 gene sequence proved to be suitable to distinguish established and supposed suppressive and nonsuppressive strains.

\section{MATERIALS AND METHODS}

Plant material. Accessions 1/93Vin and 12/93 were transmitted via dodder (Cuscuta europaea) bridges from apple to periwinkle. By the following dodder transmission from 1/93-infected periwinkle to $N$. occidentalis, strain 1/93Tab was obtained (21). Strains AT and AP15 were dodder transmitted from symptomatic apple trees to periwinkle by Marwitz et al. (12) and Carraro et al. (2), respectively. The periwinkle- and tobacco-transmitted strains were maintained in an insect-proof greenhouse by periodic grafting. Accessions 2/4, 2/7, 3/2, 3/3, 3/5, 8/7, 1/93, 12/93, $13 / 93$, and $17 / 93$ were maintained in apple trees for 10 years in the field and, following graft transmission, for at least 3 years in an unheated greenhouse. Symptomatology of these trees was assessed annually using a scoring scheme ranging from 0 (no symptoms) to 3 (severe symptoms) (23). Additional information on origin, virulence, and molecular features of the apple-maintained accessions has been provided previously $(20,21,23,24)$.

Inoculation and sampling of periwinkle. The experiments with periwinkle were carried out using genetically uniform, cutting-propagated periwinkle plants maintained in the greenhouse. Inoculation of 6 to 8 -month-old plants was performed by cleft grafting $\approx 3$-cm-long segments of diseased shoots onto the recipient plants. Each plant was graft-inoculated at three evenly distributed sites with mild strain 1/93Vin used as suppressor and at three other evenly distributed sites with either aggressive strains AT, AP15, or 12/93 used as challengers. Four trials (CP1 to CP4) were carried out. In CP1, 8 or 10 plants were first inoculated with either strain 1/93Vin or strain 12/93 and, 4 months later, were reciprocally inoculated with $12 / 93$ or $1 / 93 \mathrm{Vin}$, respectively. In $\mathrm{CP} 2$ and $\mathrm{CP} 3$, inoculation of suppressor 1/93Vin was carried out either 2.5 or 4 months prior to the inoculation of challengers AT or AP15 (cross-protection modus) or strains 1/93 Vin and AT were co-inoculated. This co-inoculation was performed at the same time as the challenger was grafted to the plants that were treated by cross protection. In trial $\mathrm{CP} 4$, only plants inoculated in the cross-protection modus were examined. In each trial, three control plants were singly inoculated with the strains included in the respective experiment. In addition, three healthy controls were included in each trial.

Following the second inoculation or co-inoculation, the plants of short-term trial $\mathrm{CP} 1$ were monitored over 6.5 months and the plants of $\mathrm{CP} 2$ to $\mathrm{CP} 4$ over 33 to 43 months for symptom development and the presence of suppressor and challengers. In each trial, usually at least three samples were randomly collected at regular intervals from shoots grown at equal distance from both the suppressor and the challenger grafting site (random shoots) and from the roots from each plant at each sampling date, as specified in the Results section. In addition, in trials CP1 and $\mathrm{CP} 4$, three samples from each plant were taken from the new growth of the scions of the secondary inoculation at each sampling date. In trial CP1, lateral shoots grown directly below this grafting site were examined as well. Furthermore, three samples from the singly inoculated control plants were regularly collected.

Inoculation and sampling of $\boldsymbol{N}$. occidentalis. Plants grown from seed were used in two trials (CPT-1 and CPT-2) carried out in the cross-protection modus. In each trial, one major shoot of seven 8-week-old plants was inoculated with suppresser 1/93Tab. In both trials, a second major shoot was inoculated with challenger strain AT 3 or 6 weeks later, respectively. In CPT-1 and CPT-2, sampling started 4 and 6 weeks, respectively, after challenging and was repeated three times in the following 3 months. From each plant, three samples were randomly collected from shoots grown at equal distance from the grafting sites of both the suppressor and the challenger. Two samples were taken from the growth of strain AT scions and from lateral shoots grown below the grafting site of the challenger. In trial CPT-1, three root samples were randomly collected from each plant at the final sampling date. At each sampling time, one shoot sample from three singly infected controls was collected.

DNA extraction and real-time polymerase chain reaction amplification. DNA from $250 \mathrm{mg}$ of roots and leaf midribs, or from shoot tips when only very small leaves were available, was extracted using a cetyltrimethylammonium bromide (CTAB) procedure (23). Toward the end of the trials, when polymerase chain reaction (PCR) amplification was inhibited in CTAB extracts from periwinkle roots, root DNA was extracted using the innuPREP Plant DNA kit (Analytik Jena, Berlin, Germany). In both procedures, DNA was recovered in $100 \mu$ of low Tris-EDTA buffer (10 mM Tris-HCl and 0.1 mM EDTA, pH 8.0). All samples from different plants and different tissues were processed individually and were never pooled.

The presence of suppressors and challengers was monitored employing multiplex real-time PCR assays using Bio-Rad iCyclers IQ or CFX 96 Touch. Variable regions of the genes encoding HflB protease AP464 and ATPases AP382 and AP460 of ' $\mathrm{Ca}$. P. mali' were selected as targets for specific detection of strains $1 / 93$ Vin, 1/93Tab, AT, AP15, and 12/93. In the trials with periwinkle, a 161-bp fragment of gene AP464 was amplified with primers fAP464-RT/rAP464-RT1. The targets of strains 1/93Vin and AT were specifically detected using Cy5/BHQ3- and FAM/BHQ1-labeled TaqMan probes 464-1/93Vin and 464ATVin, respectively. For the differential detection of strains 1/93Vin, 12/93, and AP15, 228- to 231-bp fragments of gene AP460 were amplified using primers f/rAP460-RT in combination with strain-specific TaqMan probes 460-1/93Vin and 460AP15 labeled with Cy5/BHQ3 and FAM/BHQ1, respectively (Table 1). For detection of suppressor 1/93Tab and challenger AT in $N$. occidentalis, 157- to 169-bp fragments were amplified with primer pair f/rAP382-RT. Strains 1/93Tab and AT targets were specifically detected with Cy5/BHQ3- and FAM/BHQ1-labeled TaqMan probes AP382-1/93Tab and AP382-ATnew, respectively (Table 1). The PCR reaction mixture contained the following components in a final volume of $25 \mu \mathrm{l}: 1.25 \mathrm{U}$ of TEMPase hot start Taq polymerase (Ampliqon), $1 \times$ polymerase buffer (Ampliqon II) adjusted to $2.5 \mathrm{mM} \mathrm{MgCl}_{2}, 0.2 \mathrm{mM}$ dNTPs, $0.4 \mu \mathrm{M}$ each primer, $0.2 \mu \mathrm{M}$ each probe, and $1.0 \mu \mathrm{l}$ of DNA template. The parameters used for amplification were $15 \mathrm{~min}$ at $95^{\circ} \mathrm{C}$ followed by a two-step protocol consisting of 40 cycles at $95^{\circ} \mathrm{C}$ for $20 \mathrm{~s}$ and $53^{\circ} \mathrm{C}$ for $40 \mathrm{~s}$. Samples were run in duplicates. 
Cloning, SSCP, and sequence analysis. DNA from periwinkle and $N$. occidentalis was isolated as described above. From infected apple trees, extraction was performed from phloem preparations of the current season's shoots using the CTAB procedure, according to Seemüller and Schneider (23). A fragment consisting of a variable 319- to 322-bp fragment of the 5' terminus of gene AP460 and a homogenous 136-bp fragment of the adjacent intergenic region was amplified using primer pair fAP460/rAP460-RT (Table 1). Using the components described above, the reaction was, after initial denaturation at $95^{\circ} \mathrm{C}$ for $5 \mathrm{~min}$, cycled 35 times for $1 \mathrm{~min}$ at $95^{\circ} \mathrm{C}, 45 \mathrm{~s}$ at $53^{\circ} \mathrm{C}$, and $1 \mathrm{~min}$ at $68^{\circ} \mathrm{C}$. The final step was $5 \mathrm{~min}$ at $68^{\circ} \mathrm{C}$. PCR products were ligated into pGEM-T Easy vector system (Promega, Madison, WI) and were used to transform Escherichia coli XL1 Blue cells (Stratagene, La Jolla, CA). Colony PCR was employed to amplify the inserts of recombinant plasmids using the PCR primers. The PCR products were used in SSCP analysis, as previously described (19). For sequencing of the PCR products, the recombinant plasmid DNA was extracted using a miniprep kit (Qiagen, Hilden, Germany). The cloned inserts were sequenced using M13 primers. The 319- to 322-bp sequences of the $5^{\prime}$ fragments of gene AP460 are deposited at EMBL Nucleotide Sequence Database under the accession numbers HG416936 to HG416955. Alignment of sequences and phylogenetic analysis were conducted in MEGA4 using the neighbor-joining parameters and the bootstrap test. The trees were drawn to scale, with branch lengths calculated using the average pathway method (28).

\section{RESULTS}

Symptomatology in periwinkle. The inoculated control plants differed considerably in symptomatology. Suppressor strain
1/93Vin induced only mild symptoms. Four months postinoculation (p.i.), infected plants showed mild foliar yellowing, nearly normal-sized and only weakly undulated leaves, and flowers slightly smaller than normal. During the following 8 months, symptoms slightly increased in severity but flowering remained over the entire observation periods. At termination of trials CP2 to $\mathrm{CP} 4$, the plants did not differ from healthy controls. In contrast, strains AT, AP15, and 12/93 showed much more pronounced symptoms by inducing earlier and more severe yellowing symptoms, strongly undulated and distinctly smaller leaves, and much smaller flowers than 1/93Vin. Flowering of AT- and AP15-inoculated plants ceased 8 to 10 months p.i. At the end of the observation periods, all AT- and AP15-inoculated plants were either dead or declining (Fig. 1A and B).

In CP2 to CP4, periwinkle plants first inoculated with strain 1/93Vin and challenged 2.5 to 4 months later by inoculation with strains AT or AP15 developed predominantly the mild symptoms of strain 1/93Vin (Fig. 1A). Only the growth developed from the challenger scions and, sometimes, leaves of the grafted shoot below the grafting site showed the typical symptoms induced by strains AT and AP15. These symptoms persisted for $\approx 10$ months and then slowly disappeared. Sixteen months after the secondary inoculation, no AT- or AP15-specific symptoms could be observed. Flowering of the cross-protected plants remained until the end of observation periods, as described for the plants inoculated with $1 / 93$ Vin alone. In addition, the overall symptomatology was similar (Fig. 1B). The symptomatology of the plants co-inoculated with suppressor and the challengers did not differ significantly from that of cross-protected plants. However, foliar AT symptoms, mainly on the growth of the challenger scions, persisted for up to 2 years p.i. At the end of the observation periods, no differences between healthy controls, inoculated

TABLE 1. Primers and TaqMan probes based on 'Candidatus Phytoplasma mali' gene AP382, AP460, and AP464 sequences

\begin{tabular}{|c|c|c|}
\hline Primer $(\mathrm{f} / \mathrm{r})$ or probe & DNA sequence $\left(5^{\prime}-3^{\prime}\right)$ & Strain specificity ${ }^{\mathrm{a}}$ \\
\hline fAP382-RT & CAATTGATACAGTTATTTTTGGTCCT & All strains \\
\hline rAP382-RT & TGAACAGGCCCTCTTTGTTC & All strains \\
\hline AP382-ATnew & CAGCATTAGAAAATAACGACAAAAAA & AT \\
\hline AP382-1/93Tab & TAGAAAAAAACAACGAAGACGACA & $1 / 93 \mathrm{Tab}$ \\
\hline fAP460 & TCGCCAAATAAATAATTCCCTA & All strains \\
\hline fAP460-RT & GTTATTCAATAAAGAAAAAAAATGTGC & All strains \\
\hline rAP460-RT & CAATATTTTGATAATCTTCCGGATTT & All strains \\
\hline 460-1/93Vin & GATAAAATTTACССCTTCAAAAACTG & 1/93Vin \\
\hline 460-AP15Vin & TAACTGATGCTCCAGAAGGAAAA & AP15, 12/93 \\
\hline fAP464-RT & AAATTAAATGCTCCTTGTGTGTT & All strains \\
\hline rAP464-RT1 & ATCACAATAATACCTTGAGATGG & All strains \\
\hline 464-1/93Vin & CTAGGTGGTAAAAGAGGTGGT & 1/93Vin \\
\hline 464-ATVin & TTAGGTGGTCGAAGAGGAGGA & $\mathrm{AT}$ \\
\hline
\end{tabular}

${ }^{a}$ For the respective gene.
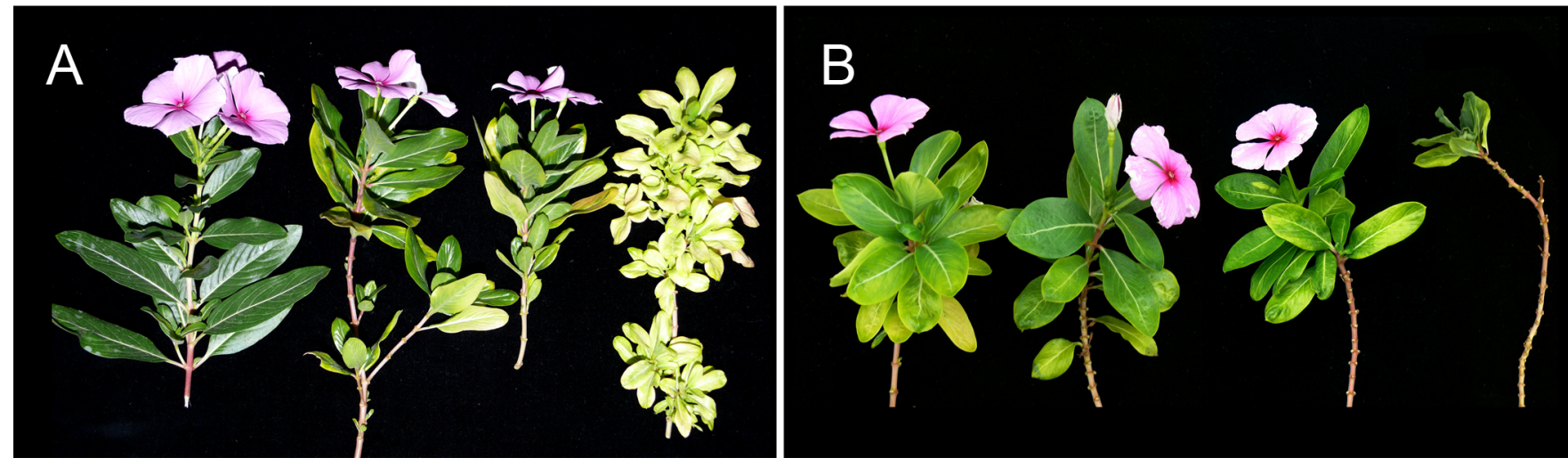

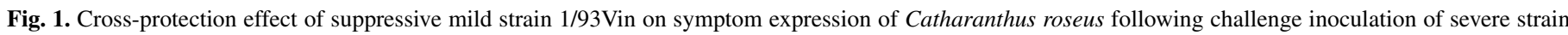

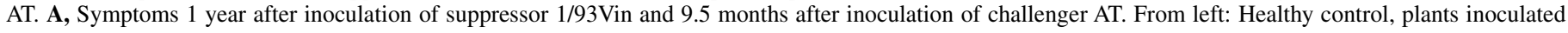

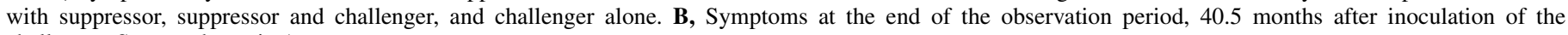
challenger. Same order as in A. 
1/93Vin controls, cross-protected, and co-inoculated plants were observed. There was no decline of cross-protected or coinoculated plants (Fig. 1A and B).

Detection of suppressor and challengers in periwinkle. In the reciprocal inoculation trial CP1, suppressive strain 1/93Vin and severe strain 12/93 responded differently. The suppressive action of $1 / 93$ Vin was evident when first or second inoculated (Table 2). In plants first inoculated with 1/93Vin and then with strain 12/93, detection rate and titer of $1 / 93 \mathrm{Vin}$ in the various sample categories remained at the same high level. Only in the new growth of the 12/93 scions was the 1/93Vin titer lower, although it tended to increase with time. In contrast, both detection rate and titer of $12 / 93$ in the growth of these scions decreased. In all other parts of the plants, strain 12/93 was not detected or the titer was close to the lower detection limit. However, strain 12/93 was detected longer in randomly collected shoots than challengers AT in CP3 and CP4 and AP15 in CP4 (see below). In plants first inoculated with 12/93 and later with $1 / 93$ Vin, detection rate and titer of $12 / 93$ in randomly collected shoots and in the roots were high throughout the observation period. In addition, all samples collected below the 1/93 inoculation site were $12 / 93$ positive, although the titer decreased. In contrast, the titer of strain 12/93 in the growth of the 1/93Vin scion was always low. Conversely, strain 1/93Vin spread into all parts of the plants, reaching detection rates of $100 \%$ or nearly $100 \%$ while the titer tended to increase. The titer of 1/93Vin in the growth of $1 / 93$ scions remained constantly high (Table 2).

In CP2, the first long-term trial, all randomly collected shoot samples tested positive for suppressor 1/93Vin 8 months after challenging. In contrast, challenger strain AT was not detectable (Table 3). This pattern persisted until the end of the trial. Similarly, strain 1/93Vin was detected in all root samples over the entire collection period whereas strain AT was detected only in a few root samples at the first two sampling dates. The figures obtained following co-inoculation differed significantly from that of cross-protected plants. At the first sampling date, detection frequency in stem samples was similar for suppressor and challenger. Then, detection rate of $1 / 93$ Vin steadily increased, reaching 100\% 27 months p.i. In contrast, detection of the challenger successively decreased but remained positive in $13 \%$ of the samples at the end of the trial. In the roots of co-inoculated plants, suppressor and challenger were at first detected in all samples. This was true for the suppressor until the end of the observation period whereas detection of the challengers decreased to $27 \%$ (Table 3).

Trial CP3 differs from CP2 by the later inoculation of the challenger (4 versus 2.5 months) and by an earlier beginning of sampling. As described in $\mathrm{CP} 2$ for cross-protected plants, strain $1 / 93$ Vin was detected in all stem and root samples, whereas AT was not detected in the stem and rarely in the roots (Table 4). Thus, these results are similar to those of $\mathrm{CP} 2$ with the shorter period before challenging. However, there were differences following simultaneous inoculation in that the detection rate of suppressor and challenger in the stem started at a higher level than in $\mathrm{CP} 2$ and the detection of the challenger in the roots at a lower level. However, at the end of $\mathrm{CP} 3$, the results were similar to those in $\mathrm{CP} 2$ except that, in $\mathrm{CP} 3$, challenger AT was not detectable in the shoots following simultaneous inoculation toward the end of the monitoring period, despite the higher detection frequency at the beginning of the monitoring.

In $\mathrm{CP} 4$, only cross-protected plants were included. This trial differs from CP2 and CP3 by the fact that the growth of the AT challenger scions was examined and that strain AP15 was included as a second challenger. As in CP2 and CP3, suppressor

TABLE 2. Presence and titer of suppressive strain 1/93Vin and severe strain 12/93 of 'Candidatus Phytoplasma mali' in randomly collected shoots, growth of secondary inoculation scions, shoots below secondary inoculation sites, and roots of periwinkle following reciprocal inoculation in trial CP1 ${ }^{\mathrm{a}}$

\begin{tabular}{|c|c|c|c|c|c|c|c|c|c|c|c|c|c|c|c|c|}
\hline \multirow[b]{3}{*}{ Months ${ }^{b}$} & \multicolumn{4}{|c|}{ Random shoots } & \multicolumn{4}{|c|}{ Secondary scions } & \multicolumn{4}{|c|}{ Below secondary inoculation site } & \multicolumn{4}{|c|}{ Roots } \\
\hline & \multicolumn{2}{|c|}{ 1/93Vin } & \multicolumn{2}{|c|}{$12 / 93$} & \multicolumn{2}{|c|}{ 1/93Vin } & \multicolumn{2}{|c|}{$12 / 93$} & \multicolumn{2}{|c|}{ 1/93Vin } & \multicolumn{2}{|c|}{$12 / 93$} & \multicolumn{2}{|c|}{ 1/93Vin } & \multicolumn{2}{|c|}{$12 / 93$} \\
\hline & Det & $\mathrm{Ct}$ & Det & $\mathrm{Ct}$ & Det & $\mathrm{Ct}$ & Det & $\mathrm{Ct}$ & Det & $\mathrm{Ct}$ & Det & $\mathrm{Ct}$ & Det & $\mathrm{Ct}$ & Det & $\mathrm{Ct}$ \\
\hline 3.5 & $8 / 8$ & 20.6 & $7 / 8$ & 32.8 & $16 / 16$ & 25.7 & $16 / 16$ & 21.0 & $15 / 16$ & 21.9 & $10 / 16$ & 33.3 & $16 / 16$ & 22.9 & $1 / 16$ & 35.1 \\
\hline 4.5 & $8 / 8$ & 20.3 & $8 / 8$ & 33.0 & $24 / 24$ & 24.1 & $20 / 24$ & 26.0 & $24 / 24$ & 21.6 & $15 / 24$ & 34.1 & $16 / 16$ & 23.1 & $3 / 16$ & 34.8 \\
\hline 6.5 & $8 / 8$ & 22.2 & $2 / 8$ & 35.8 & $23 / 24$ & 23.4 & $13 / 24$ & 31.7 & $24 / 24$ & 23.5 & $8 / 24$ & 35.0 & $16 / 16$ & 23.9 & $4 / 16$ & 35.1 \\
\hline $12 / 93$ & & & & & & & & & & & & & & & & \\
\hline 6.5 & $10 / 10$ & 29.9 & $10 / 10$ & 20.2 & $29 / 30$ & 22.9 & $15 / 30$ & 31.6 & $30 / 30$ & 25.5 & $26 / 30$ & 24.8 & $20 / 20$ & 23.1 & $20 / 20$ & 22.3 \\
\hline
\end{tabular}

a Trial CP1 = secondary inoculation of strains 1/93Vin and 12/93 4 months after inoculation of the primary strain. Det = detection: number of polymerase chain reaction (PCR)-positive samples/number of samples examined. $\mathrm{Ct}=$ mean cycle threshold value of PCR-positive samples. Values $>36.0$ or N/A (no amplification) within 40 cycles of real-time PCR were considered negative.

${ }^{\mathrm{b}}$ Sampling time post secondary inoculation. $1 / 93$ Vin = primary inoculation1/93Vin, secondary inoculation 12/93 and 12/93= primary inoculation12/93, secondary inoculation 1/93Vin.

TABLE 3. Detection of suppressive strain 1/93Vin and challenger strain AT of 'Candidatus Phytoplasma mali' in trial CP2 in randomly collected shoots (S) and roots $(\mathrm{R})$ of periwinkle following inoculation of suppressor and challenger in the cross-protection modus or simultaneously ${ }^{\mathrm{a}}$

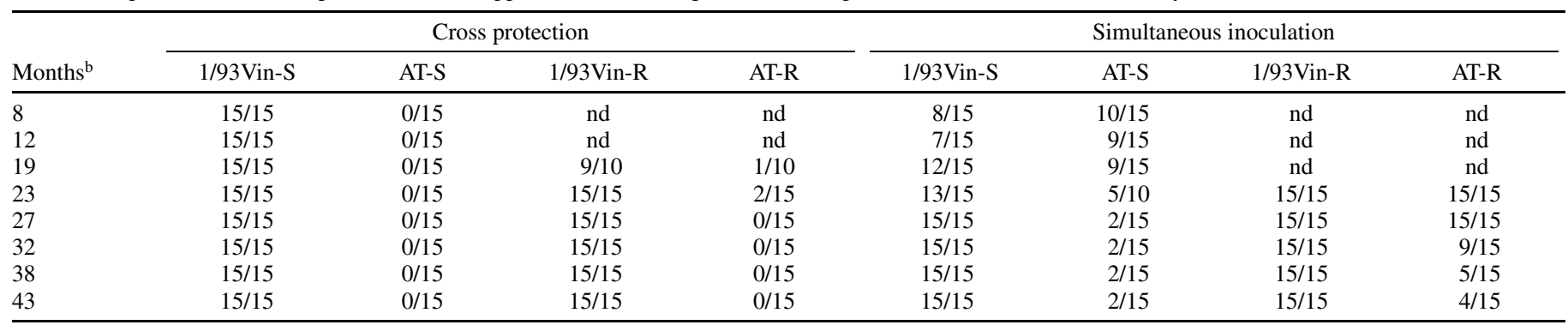

${ }^{a}$ Inoculation of challenger and simultaneous inoculation 2.5 months after primary inoculation of suppressor. Numbers shown indicate number of polymerase chain reaction-positive samples/number of samples examined; $\mathrm{nd}=$ not determined.

b Sampling postinoculation of challenger or simultaneous inoculation. 
1/93Vin was detected in all randomly collected stem samples and in all root samples, whereas strain AT was not detected in randomly collected stem samples (Table 5). However, the challenger was detected in a few root samples throughout the observation period. In the growth of the strain AT scions, the suppressor was detected in $60 \%$ of the samples of the challenger 4 months p.i. Then, detection rate increased, reaching $100 \% 8$ months later. In the same samples, detection frequency of strain AT was first $100 \%$ and then decreased to 0 over a period of 28 months. Challenger AP15, of which only stem samples were examined, was never detected and, thus, responded like challenger AT in trials CP2 through CP4.

Symptomatology of $N$. occidentalis. $N$. occidentalis was considerably more sensitive to phytoplasma infection than periwinkle. Even suppressor 1/93Tab, which proved to be largely avirulent in apple trees (see above), induced distinct symptoms consisting of foliar yellowing associated with enlargement and browning of veins. With the development of foliar symptoms, flower size decreased and flowering ceased 2 to 3 months p.i.
However, rudimentary flower structures remained. The plants declined 4 to 5 months p.i. Symptomatology was similar on plants primarily inoculated with 1/93Tab and challenged with strain AT. Symptoms of plants inoculated with strain AT alone were more pronounced, leading to decline 1 month earlier than plants cross protected with 1/93Tab. Healthy controls grew vigorously and were flowering throughout the observation periods.

Detection of suppressor and challenger in $N$. occidentalis. The results obtained in the two cross-protection experiments correspond well with those of the cross-protection trials of CP2 to CP4 with periwinkle. In both trials, suppressor 1/93Tab was detected in all randomly collected stem samples whereas challenger AT was never detected in these samples (Table 6). In addition, the suppressor was detected in all 21 root samples collected in CPT-1 at the end of the experiment whereas the tests were negative for the challenger (data not shown). In contrast, the challenger was detected in the new growth of the AT inoculation scion and in lateral shoots below the AT grafting side. However, there were distinct differences between the two trials, due to the

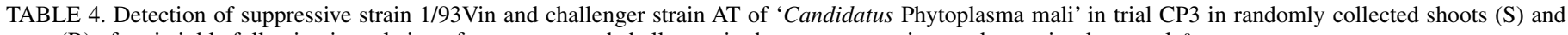
roots $(\mathrm{R})$ of periwinkle following inoculation of suppressor and challenger in the cross-protection modus or simultaneously ${ }^{\mathrm{a}}$

\begin{tabular}{|c|c|c|c|c|c|c|c|c|}
\hline \multirow[b]{2}{*}{ Months $^{\mathrm{b}}$} & \multicolumn{4}{|c|}{ Cross protection } & \multicolumn{4}{|c|}{ Simultaneous inoculation } \\
\hline & 1/93Vin-S & AT-S & 1/93Vin-R & AT-R & 1/93Vin-S & AT-S & 1/93Vin-R & AT-R \\
\hline 3.5 & $15 / 15$ & $0 / 15$ & nd & nd & $12 / 15$ & $15 / 15$ & nd & nd \\
\hline 8 & $15 / 15$ & $0 / 15$ & nd & nd & $14 / 15$ & $11 / 15$ & nd & nd \\
\hline 12 & $15 / 15$ & $0 / 15$ & $15 / 15$ & $0 / 15$ & $15 / 15$ & $5 / 35$ & $15 / 15$ & $8 / 15$ \\
\hline 16 & $15 / 15$ & $0 / 15$ & $15 / 15$ & $0 / 15$ & $15 / 15$ & $2 / 15$ & $12 / 15$ & $4 / 15$ \\
\hline 21 & $15 / 15$ & $0 / 15$ & $15 / 15$ & $0 / 15$ & $15 / 15$ & $0 / 15$ & $15 / 15$ & $3 / 15$ \\
\hline 27 & $15 / 15$ & $0 / 15$ & $15 / 15$ & $0 / 15$ & $15 / 15$ & $0 / 15$ & $15 / 15$ & $3 / 15$ \\
\hline 33 & $15 / 15$ & $0 / 15$ & $15 / 15$ & $1 / 15$ & $15 / 15$ & $0 / 15$ & $15 / 15$ & $3 / 15$ \\
\hline
\end{tabular}

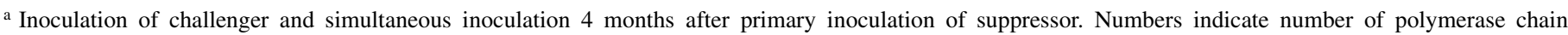
reaction-positive samples/number of samples examined; nd = not determined.

b Sampling postinoculation of challenger or simultaneous inoculation.

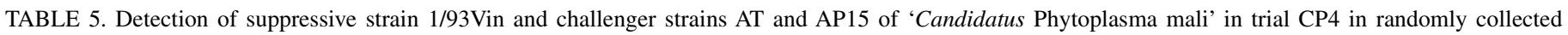
shoots (S) and growth of the AT inoculation scion (AT-SC) and roots (R) of periwinkle following cross-protection inoculation ${ }^{\mathrm{a}}$

\begin{tabular}{|c|c|c|c|c|c|c|c|}
\hline Sampling (months) ${ }^{\mathrm{b}}$ & 1/93Vin-S & AT-S & 1/93Vin_AT-SC & AT_AT-SC & 1/93Vin-R & AT-R & AP15-S \\
\hline 4 & $15 / 15$ & $0 / 15$ & $9 / 15$ & $15 / 15$ & nd & nd & $0 / 10$ \\
\hline 8 & $15 / 15$ & $0 / 15$ & $11 / 15$ & $11 / 15$ & nd & nd & $0 / 10$ \\
\hline 12 & $15 / 15$ & $0 / 15$ & $15 / 15$ & $6 / 15$ & $15 / 15$ & $1 / 10$ & $0 / 10$ \\
\hline 16 & $15 / 15$ & $0 / 15$ & $15 / 15$ & $4 / 15$ & $15 / 15$ & $1 / 10$ & $0 / 10$ \\
\hline 21 & $15 / 15$ & $0 / 15$ & $15 / 15$ & $3 / 15$ & $15 / 15$ & $1 / 10$ & $0 / 10$ \\
\hline 26 & $15 / 15$ & $0 / 15$ & $15 / 15$ & $2 / 15$ & $15 / 15$ & $2 / 10$ & $0 / 10$ \\
\hline 32 & $15 / 15$ & $0 / 15$ & $15 / 15$ & $0 / 15$ & $15 / 15$ & $2 / 10$ & $0 / 10$ \\
\hline 38 & $15 / 15$ & $0 / 15$ & $15 / 15$ & $0 / 15$ & $15 / 15$ & $1 / 10$ & $0 / 10$ \\
\hline
\end{tabular}

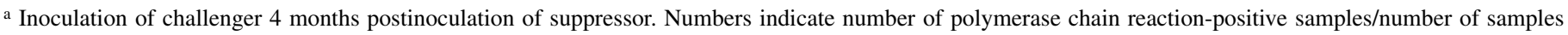
examined; nd = not determined.

b Postinoculation of challenger or simultaneous inoculation.

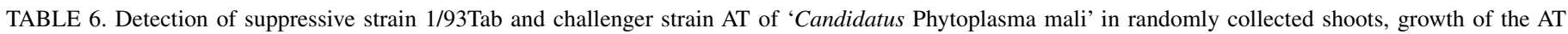
inoculation scions and lateral shoots below the AT grafting site of Nicotiana occidentalis following cross-protection inoculation in trials CPT- 1 and CPT- $2^{\text {a }}$

\begin{tabular}{|c|c|c|c|c|c|c|}
\hline \multirow[b]{2}{*}{ Sampling (weeks) ${ }^{\mathrm{b}}$} & \multicolumn{2}{|c|}{ Random shoots } & \multicolumn{2}{|c|}{ AT scion } & \multicolumn{2}{|c|}{ Below AT scion } \\
\hline & 1/93Tab & AT & 1/93Tab & $\mathrm{AT}$ & 1/93Tab & AT \\
\hline \multicolumn{7}{|l|}{ СРТ-1 } \\
\hline 6 & $21 / 21$ & $0 / 21$ & $4 / 14$ & $10 / 14$ & $10 / 14$ & $4 / 14$ \\
\hline 8 & $21 / 21$ & $0 / 21$ & $7 / 14$ & $7 / 14$ & $13 / 14$ & $1 / 14$ \\
\hline 10 & $21 / 21$ & $0 / 21$ & $13 / 14$ & $1 / 14$ & $14 / 14$ & $0 / 14$ \\
\hline 13 & $21 / 21$ & $0 / 21$ & $14 / 14$ & $0 / 14$ & $14 / 14$ & $0 / 14$ \\
\hline \multicolumn{7}{|l|}{ СРТ-2 } \\
\hline 6 & $21 / 21$ & $0 / 21$ & $12 / 14$ & $2 / 14$ & $13 / 14$ & $1 / 14$ \\
\hline 8 & $21 / 21$ & $0 / 21$ & $14 / 14$ & $0 / 14$ & $14 / 14$ & $0 / 14$ \\
\hline 10 & $21 / 21$ & $0 / 21$ & $14 / 14$ & $0 / 14$ & $14 / 14$ & $0 / 14$ \\
\hline 13 & $21 / 21$ & $0 / 21$ & $14 / 14$ & $0 / 14$ & $14 / 14$ & $0 / 14$ \\
\hline
\end{tabular}

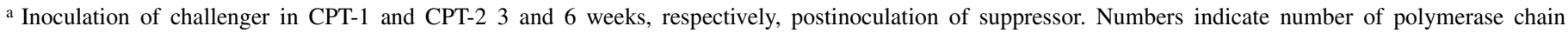
reaction-positive samples/number of samples examined.

b Postinoculation of challenger. 
different incubation period of the suppressor of 3 versus 6 weeks in trials CPT-1 and CPT-2, respectively, prior to challenge inoculation. These differences are evident in a much higher detection rate of the suppressor and a much lower detection rate of the challenger in the growth of the AT scion in CPT-2. Differences in the detection of suppressor and challenger in the lateral shoots below the AT grafting site were less pronounced in the two trials but showed the same tendency. Despite these differences, the suppressor was detected in all samples by the end of the experiments whereas detection of the challenger decreased to zero in both experiments.

Pathogenic and molecular features of suppressive strains. SSCP analysis of cloned gene AP460 fragments of $\approx 455$ bp in length amplified with primers fAP460/rAP460-RT revealed that suppressive strains $1 / 93 \mathrm{Vin}$ and 1/93Tab show similar profiles consisting of one group of bands. These profiles are distinctly different from those of aggressive periwinkle- and $N$. occidentalis-maintained challenger strains AT, AP15, and 12/93 that are composed of two groups of bands. Both types of profiles were also identified in cloned PCR fragments of AP phytoplasma strains maintained in apple (Fig. 2). In order to relate the presence of these markers in infected apple trees to phytopathogenic data, the disease history of apple trees infected with various accessions and grown in the field and subsequently in an unheated greenhouse was examined. This analysis showed that the symptomatology of the trees differs considerably in both the field-growing and the greenhouse-growing period as well as between these two phases. Except for accession 17/93, all accession showed witches'-broom as a severe symptom once or repeatedly in the field-growing or the greenhouse growing period and all of them

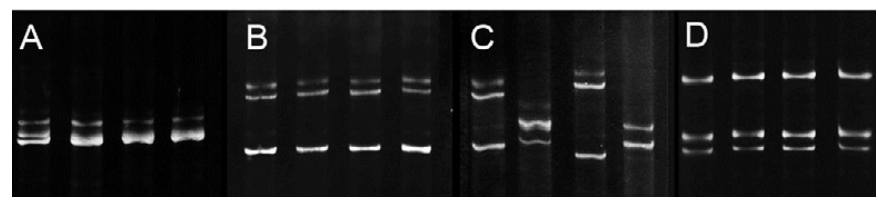

Fig. 2. Single-strand conformation polymorphism (SSCP) profiles of DNA fragments of the $5^{\prime}$ end of gene AP460 and the adjacent spacer region of 'Candidatus Phytoplasma mali' strains. From left: A, Suppressive mild strains 1/93Vin, 1/93Tab from periwinkle and Nicotiana occidentalis, and two samples from symptomless apple tree infected by multiple-strain accession $1 / 93$; B, samples from diseased, 1/93-infected apple tree showing profiles of nonsuppression; $\mathbf{C}$, samples from slightly diseased, 1/93-infected apple tree showing profiles associated with both suppression and nonsuppression; D, samples from nonsuppressive severe strains AT and AP15 from periwinkle and $12 / 93$ and $3 / 5$ from diseased apple trees. did not show symptoms for shorter of longer periods. This typical disease history for AP indicates shifts in the predominance of mild and severe phytoplasma strains. The symptomatology at sampling corresponds well not only with strain virulence but also with the prevalence of strains possessing the markers of gene 460 for suppression or nonsuppression. In accessions inducing severe symptoms, only cloned fragments showing the profile of nonsuppressive strains were identified whereas, in symptomless trees, only suppression-associated clones were observed. Both types of fragments were detected in trees exhibiting mild symptoms. An exception to this pattern is accession $3 / 3$ that was symptomless at sampling and in the previous 5 years and exhibited the nonsuppressive profile. This pattern may indicate the presence of a nonsuppressive mild strain (Table 7).

Based on the SSCP profiles, gene AP460 inserts of recombinant plasmids were sequenced and the variable 319 - to 322-bp 5' fragments used in phylogenetic analysis. Construction of the phylogram resulted in two homogenous major branches represented by fragments of suppressive strains 1/93 Vin and 1/93Tab and by the putatively nonsuppressive strains AT, AP15, and 12/93 (Fig. 3). The suppression-associated sequences of mild strains, including those of accession 1/93 from apple, periwinkle, and $N$. occidentalis and several other accessions from apple, were identical or nearly identical and cluster in one major branch. In contrast, sequences of severe strains of the 1/93 complex and of accessions $3 / 2$ and 13/93, which are also composed of mild suppressive and severe nonsuppressive strains, cluster with other severe strains in the second major branch. Mild strain 3/3 differs from this scheme by clustering with severe and nonsuppressive strains. The separate clustering of suppressive strains is mainly due to 14 unique substitutions and the deletion of codon CAA in the 150-bp segment starting downstream of the transmembraneencoding region and extending to position 286 of the cloned AP460 gene fragment. These differences result in the deletion of a Gln residue and in nine altered gene products.

\section{DISCUSSION}

Observations on antagonistic interferences of phytoplasma strains have been published since the middle of last century. From their work with aster yellows (' $\mathrm{Ca}$. P. asteris') agents and based on symptomatology, Kunkel (10) and Freitag (5) reported on mutual suppression of closely related strains. In plants inoculated in the cross-protection modus, the primary phytoplasmas usually suppressed challenging strains inoculated 1 or 2 weeks later. Less often, the replacement of an existing strain by the challenger was

TABLE 7. Correlation of suppression-associated single-strand conformation polymorphism (SSCP) profiles of a DNA fragment of the 5 ' region of AAA+ ATPase gene AP460 and an adjacent spacer segment with the symptoms induced by 'Candidatus Phytoplasma mali' accessions in apple

\begin{tabular}{|c|c|c|c|c|c|}
\hline \multirow[b]{2}{*}{ Accession } & \multicolumn{3}{|c|}{ Symptoms, virulence ${ }^{a}$} & \multicolumn{2}{|c|}{ SSCP profiles ${ }^{b}$} \\
\hline & 10 Years, field growing ${ }^{\mathrm{c}}$ & 3 Years, greenhouse $\mathrm{e}^{\mathrm{c}}$ & At sampling ${ }^{\text {d }}$ & Suppressive & Nonsuppressive \\
\hline $1 / 93$ & 0.2 & 0.0 & 0.0 & 121 & 0 \\
\hline $1 / 93$ & 0.2 & 3.0 & 3.0 & 0 & 59 \\
\hline $1 / 93$ & 0.2 & 0.7 & 1.5 & 43 & 22 \\
\hline $12 / 93$ & 2.4 & 3.0 & 3.0 & 0 & 13 \\
\hline $13 / 93$ & 0.8 & 0.5 & 0.2 & 156 & 1 \\
\hline $17 / 93$ & 0.1 & 0.0 & 0.0 & 43 & 0 \\
\hline $2 / 4$ & 1.7 & 0.2 & 0.0 & 82 & 0 \\
\hline $2 / 7$ & 0.0 & 1.5 & 3.0 & 0 & 27 \\
\hline $3 / 1$ & 1.0 & 3.0 & 3.0 & 0 & 26 \\
\hline $3 / 3$ & 1.4 & 0.0 & 0.0 & 0 & 30 \\
\hline $3 / 2$ & 0.9 & 0.5 & 0.5 & 9 & 19 \\
\hline $3 / 5$ & 1.7 & 3.0 & 3.0 & 0 & 22 \\
\hline $8 / 7$ & 0.0 & 3.0 & 3.0 & 0 & 25 \\
\hline
\end{tabular}

a Rating scheme from 0 (no symptoms or virulence) to 3 (severe symptoms or virulence).

b Number of SSCP profiles indicating suppression or nonsuppression.

c Mean annual values.

d At greenhouse-growing period. 
observed as well. Another antagonistic interaction is reported to result in mutual suppression of both strains and in the development of symptomless plants. Mutual suppression in leafhopper vectors was also described and was based on the observation that the phytoplasma strain that was acquired first obviously prevented the acquisition or multiplication of a second strain when the vector was subsequently fed on a plant infected with this strain (10). Cross-protection activity was also observed between various phytoplasma types reported to be associated with potato witches'broom diseases (29).

There are also reports on phytoplasma strain interactions in infected stone fruit trees. In reciprocal cross-protection experiments with the peach yellows and little peach agents of the Xdisease phytoplasma group, Kunkel (9) found that the inoculated peach seedlings developed only the symptoms induced by the primary phytoplasma. Later, in France, it was observed that, in orchards severely affected by ' $\mathrm{Ca}$. P. prunorum'-induced apricot chlorotic leaf roll, a few trees recovered from disease and then performed as well as healthy trees, or nearly so. In subsequent inoculation experiments, it has been shown that trees inoculated by grafting scions from recovered trees were little affected or unaffected when subsequently grown at heavily infested sites where untreated trees declined. In such pretreated trees, a phytoplasma was identified that showed the same restriction fragment length polymorphism patterns of $16 \mathrm{~S}$ ribosomal DNA as severe ' $C a$. P. prunorum' strains. It was suggested that avirulent or mild strains of this phytoplasma are responsible for the crossprotection effect $(3,14)$.

Another example of suppression in closely related phytoplasmas was observed in strains of the ash yellows agent ' $\mathrm{Ca}$. P. fraxini' differing significantly in their degree of aggressiveness. When either of such periwinkle-maintained strains was inoculated 11 weeks before the other into the same plant, only the initial strain could be detected by PCR after a further 12 weeks of incubation. Thus, it has been suggested that the initial strain or its effect on the host may have interfered with multiplication or longdistance movement of the second strain (25).

From the above references, there is little or no information on the fate of the phytoplasma populations involved and their genetic characteristics. However, in previous work, we were able to distinguish and specifically amplify both mild and severe strains of the AP phytoplasma based on sequences of several AAA+ ATPase and $h f l B$ genes. Furthermore, we could show that multiple strain infections are common and that they usually consist of mild and severe strains. Under still poorly understood circumstances, shifts in the composition of the populations may occur. Because symptom expression depends on the prevailing strains, such changes determine disease appearance and severity and may result in recovery or in reappearance of severe symptoms. Recovery of AP-diseased apple trees has been defined as restriction of the phytoplasma infection to the roots and the temporary or persistent failure of the pathogen to spread to the aerial parts to induce symptoms (2). As observed in our work with accession $1 / 93$, avirulent and mild strains predominate in the roots of recovered trees whereas severe strains persist latently only in low numbers $(20,21)$.

Due to our observations on accession 1/93, a suppressive action of the mild strains was taken into consideration. The inoculation experiments with the experimental hosts periwinkle and $N$. occidentalis reported here confirmed this assumption, in that the suppressive activity of strains 1/93Vin and 1/93Tab was shown in several trials. The antagonistic action of both suppressors was most striking in the long-term trials with periwinkle and in the trials with $N$. occidentalis carried out in the cross-protection modus. In these experiments, the suppressor was identified in all randomly collected stem and all root samples over the entire observation period. In contrast, the challengers were not detected in the stem and rarely in the roots. Following simultaneous inoculation in $\mathrm{CP} 2$ to $\mathrm{CP} 4$, the suppressor successively colonized all stem and root regions whereas detection of challenger AT decreased. However, this strain usually remained detectable in up to $13 \%$ of the stem samples and $27 \%$ of the root samples. These data indicate that strains $1 / 93 \mathrm{Vin}$ and $1 / 93 \mathrm{Tab}$ are powerful suppressors that either eliminate the challengers or reduce their population below the detection level and to a concentration which did not markedly affect the plants. The results obtained, in particular by simultaneous inoculation, by examining the development of the challengers in the growth of their grafting scions and by preinoculating strain $12 / 93$ indicate that the suppression observed is influenced by but not depending on the strain inoculated first. Thus, the mechanism appears different from the primary inoculum-dependent suppression described $(10,25)$. In work by Ermacora et al. (4) with two aggressive ' $\mathrm{Ca}$. P. mali' strains, no indication of suppression was observed, regardless of the order of inoculation.

The results of this study may suggest that suppressive strains are involved in the widespread recovery phenomenon. In the leaf phloem of recovered trees, a range of ultrastructural, cytochemical, and biochemical aberrations has been observed that are supposed to lead to defense reactions and prevent stem colonization (15-18). However, the triggering mechanism of these alterations is poorly understood and there is no explanation for how a rootresiding phytoplasma population may induce systemic defense reactions in the leaves against a very closely related microbe without being affected itself. In our study, the survival rate of aggressive strain AT was higher in the root than in the stem; therefore, it is conceivable that suppressive strains inhibit multiplication of severe and nonsuppressive strains and their spread from the roots to the stem. However, the question remains of why the suppressors do not colonize the aerial parts of recovered apple trees.

As previously shown, deduced sequences of several AAA+ proteins, including AP460, from mild and severe ' $\mathrm{Ca}$. P. mali'

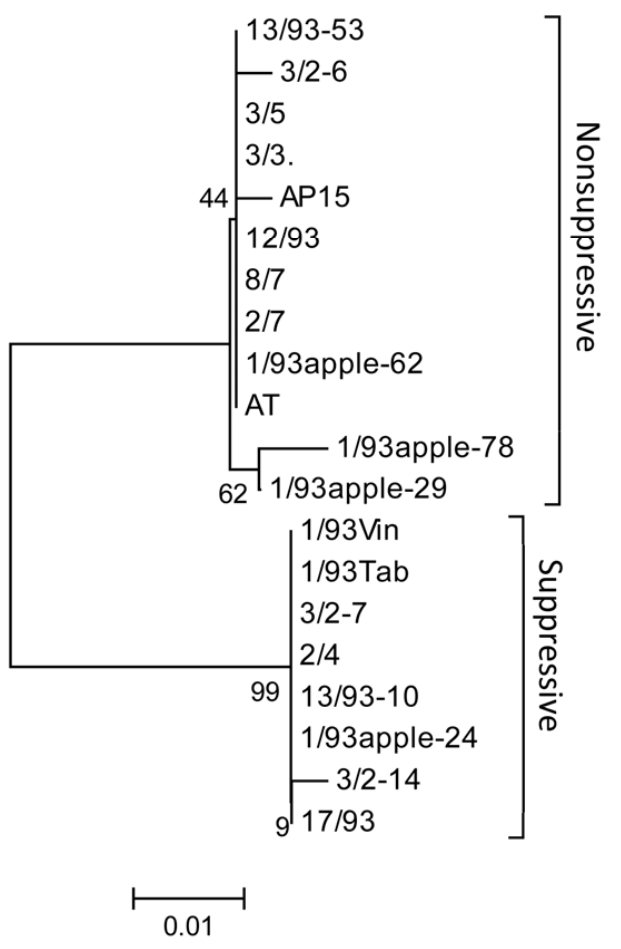

Fig. 3. Distant phylogenetic clustering of cloned 319- to 322-bp 5' fragment sequences of ATPase gene AP460 from suppressive and nonsuppressive strains of 'Candidatus Phytoplasma mali'. Extensions of accession designations indicate clone number of accessions clustering in both subclades. Only one cloned fragment is shown from accessions with identical inserts. 
strains cluster distantly, according to their virulence $(20,24)$. The distinct clustering of sequences of DNA fragments of suppressive and nonsuppressive ' $C a$. P. mali' strains as identified in the current study does not occur in other AAA+ proteins of ' $\mathrm{Ca}$. P. mali'. The close relationship of strain pathogenicity, suppressive action, and molecular data appears to indicate that ATPase AP460 is involved in suppression. Such an antagonistic action appears possible because the ATPase-carrying C-tail of transmembrane AP460 protein is predicted to face the sieve tube environment and may, in this way, become in contact with other phytoplasmas or may provide energy for reactions affecting virulent strains or triggering specific mechanisms. The identification of suppressive strains in ' $\mathrm{Ca}$. P. mali' based on the AP460 protein is another indication of the presumption that AAA+ proteins are involved in appearance and severity of $\mathrm{AP}$, as previously suggested (24).

The mode of action of phytoplasma suppression is poorly understood. It is conceivable that suppressive strains may affect other strains by occupying attachment sites or by competitive utilization of nutrition sources. In addition, production of inhibitory or toxic substances may target co-inhabiting or competing strains. From many gram-negative bacteria, there are increasing amounts of data on the effect on competing microbes based on the recently discovered type VI secretion system (T6SS). The T6SS secretory machinery includes AAA+ proteins $\mathrm{ClpB} / \mathrm{ClpV}$ and several other proteins that are encoded in a single locus. T6SS is involved in pathogenicity to the eukaryotic host and in the control of co-inhabiting or competing microbes. For instance, the T6SS of Pseudomonas syringae and Vibrio cholerae was shown to target host cells and confers toxicity toward other microbes. The range of their targets may be very wide and include protists and distantly related bacteria or may be very narrow, such as closely related strains of the same species $(6,8,11)$. It is conceivable that the AAA+ protein AP460 of suppressive strains of ' $C a$. P. mali' is part of such a system present in gram-positive bacteria and the related phytoplasmas.

In plant virology, mixed infections are common in nature and are considered to be the rule rather than the exception. Virusvirus interactions have been studied for many years and have been identified to be either antagonistic or synergistic. Research on such interactions provided evidence that they may be of crucial significance for the understanding of viral pathogenesis (27). Although a wealth of information on virus interactions is available, there are only a few examples of utilizing cross protection as a control measure. The most important example is citrus tristeza in Brazil, where cross protection by mild strains is currently the only means to protect sensitive commercial sweet orange and grapefruit cultivars against damage by severe strains of the virus (13). However, before cross protection can be recommended to control phytoplasma diseases of fruit trees and other persistent crop plants, further research is required. Of particular importance is the need for more information on range, duration, and stability of the cross-protection effect achieved by the strains used and on the factors leading to shifts in phytoplasma populations. In our study, some variation in the effect of suppressive strains was observed. The data indicate that inoculation in the cross-protection modus yields better results than co-inoculation and that the time between first and second inoculation may play a role. In addition, as shown for strain 12/93 in CP1, the genotype of the challenger may affect the results as well.

\section{ACKNOWLEDGMENTS}

The study was supported by a grant to S. Sule from the Humboldt Foundation. We thank F. Hergenhahn, V. Lessle, T. Schneider, and E. Kiss for their excellent technical assistance; J. Thompson for correcting the English text of an earlier version of the manuscript; and C. Berwarth and J. Jaworski for taking the photographs and preparing the figures.

\section{LITERATURE CITED}

1. Carraro, L., Ermacora, P., Loi, N., and Osler, R. 2004. The recovery phenomenon in apple proliferation-infected apple trees. J. Plant Pathol. 86:141-146.

2. Carraro, L., Osler, R., Refatti, E., and Poggi Pollini, C. 1988. Transmission of the possible agent of apple proliferation to Vinca rosea by dodder. Riv. Patol. Veg. Ser. 4, 24:43-52.

3. Castelain, C., Jullian, J.-P., Lemaire, J.-M., and Morvan, G. 2007. Lutte biologique contre l'ECA: la prémunition. Arboriculture 611:33-38.

4. Ermacora, P., Martini, M., Carraro, L., Ferrini, F., and Loi, N. 2007. Behaviour and interaction of two 'Candidatus Phytoplasma mali' strains in artificial infections. Bull. Insectol. 60:203-204.

5. Freitag, J. H. 1964. Interaction and mutual suppression among three strains of aster yellows virus. Virology 24:401-413.

6. Haapalainen, M., Mosorin, H., Dorati, F., Wu, R. F., Roine, E., Taira, S., Nissinen, R., Mattinen, L., Jackson, R., Pirhonen, M., and Lin, N.-C. 2012. Hcp2, a secreted protein of the phytopathogen Pseudomonas syringae pv. tomato DC3000, is required for fitness for competition against bacteria and yeasts. J. Bacteriol. 194:4810-4822.

7. Hanson, P. I., and Whiteheart, S. W. 2005. AAA+ proteins: have engine, will work. Nat. Rev. Mol. Cell Biol. 6:519-529.

8. Hood, R. D., Singh, P., Hsu, F. S., Guvener, T., Carl, M. A., Trinidad, R. R. S., Silverman, J. M., Ohlson, B. B., Hicks, K. G., Plemel, R. L., Li, M., Schwarz, S., Wan, W. Y., Merz, A. J. Goodlett, D. R., and Mougous, D. 2010. A type VI secretion system of Pseudomonas aeruginosa targets, a toxin to bacteria. Cell Host Microbe 7:25-37.

9. Kunkel, L. O. 1936. Immunological studies on the three peach diseases, yellows, rosette, and little peach. Phytopathology 26:201-219.

10. Kunkel, L. O. 1955. Cross protection between strains of aster yellows. Adv. Virus Res. 3:251-273.

11. MacIntyre, D. L., Miyata, S. T., Kitaoka, M., and Pukatzki, S. 2010. The Vibrio cholerae type VI secretion system displays antimicrobial properties. Proc. Natl. Acad. Sci. USA 107:19520-19524.

12. Marwitz, R., Petzold, H., and Özel, M. 1974. Untersuchungen zur Übertragbarkeit des möglichen Erregers der Triebsucht des Apfels auf einen krautigen Wirt. Phytopathol. Z. 81:85-91.

13. Moreno, P., Ambros, S., Albiach-Marti, M. R., Guerri, J., and Pena, L. 2008. Plant diseases that changed the world-Citrus tristeza virus: a pathogen that changed the course of the citrus industry. Mol. Plant Pathol. 9:251-268

14. Morvan, G., Castelain, C., and Arnoux, M. 1986. Prospective for the control of apricot chlorotic leaf roll, a mycoplasma disease, by cross protection. Acta Hortic. 193:359-366.

15. Musetti, R., Di Toppi, L. S., Ermacora, P., and Favali, M. A. 2004. Recovery in apple trees infected with the apple proliferation phytoplasma: an ultrastructural and biochemical study. Phytopathology 94:203208.

16. Musetti, R., Farhan, K., De Marco, F., Polizzotto, R., Paolacci, A., Ciaffi, M., Ermacora, P., Grisan, S., Santi, S., and Osler, R. 2013. Differentiallyregulated defence genes in Malus domestica during phytoplasma infection and recovery. Eur. J. Plant Pathol. 136:13-19.

17. Musetti, R., Paolacci, A., Ciaffi, M., Tanzarella, O. A., Polizzotto, R., Tubaro, F., Mizzau, M., Ermacora, P., Badiani, M., and Osler, R. 2010. Phloem cytochemical modification and gene expression following the recovery of apple plants from apple proliferation disease. Phytopathology 100:390-399.

18. Patui, S., Bertolini, A., Clincon, L., Ermacora, P., Braidot, E., Vianello, A., and Zancani, M. 2013. Involvement of plasma membrane peroxidases and oxylipin pathway in the recovery from phytoplasma disease in apple (Malus domestica). Physiol. Plant. 148:200-213.

19. Schneider, B., and Seemüller, E. 2009. Strain differentiation of 'Candidatus Phytoplasma mali' by SSCP and sequence analyses of the hflB gene. J. Plant Pathol. 91:103-112.

20. Seemüller, E., Kampmann, M., Kiss, E., and Schneider, B. 2011. HfIB gene-based phytopathogenic classification of Candidatus Phytoplasma mali strains and evidence that strain composition determines virulence in multiply infected apple trees. Mol. Plant-Microbe Interact. 24:1258-1266.

21. Seemüller, E., Kiss, E., Sule, S., and Schneider, B. 2010. Multiple infection of apple trees by distinct strains of 'Candidatus Phytoplasma mali' and its pathological relevance. Phytopathology 100:863-870.

22. Seemüller, E., Kunze, L., and Schaper, U. 1984. Colonization behavior of MLO, and symptom expression of proliferation-diseased apple trees and decline-diseased pear trees over a period of several years. Z . Pflanzenkrankh. Pflanzenschutz 91:525-532.

23. Seemüller, E., and Schneider, B. 2007. Differences in virulence and genomic features of strains of 'Candidatus Phytoplasma mali', the apple proliferation agent. Phytopathology 97:964-970.

24. Seemüller, E., Sule, S., Kube, M., Jelkmann, W., and Schneider, B. 
2013. The AAA+ ATPases and $\mathrm{HflB} / \mathrm{FtsH}$ proteases of 'Candidatus Phytoplasma mali': Phylogenetic diversity, membrane topology, and relationship to strain virulence. Mol. Plant-Microbe Interact. 26:367376.

25. Sinclair, W. A., and Griffiths, H. M. 2000. Variation in aggressiveness of ash yellows phytoplasmas. Plant Dis. 84:282-288.

26. Snider, J., Thibault, G., and Houry, W. A. 2008. The AAA plus superfamily of functionally diverse proteins. Genome Biol. 9:216.
27. Syller, J. 2012. Facilitative and antagonistic interactions between plant viruses in mixed infections. Mol. Plant Pathol. 13:204-216.

28. Tamura, K., Dudley, J., Nei, M., and Kumar, S. 2007. MEGA4: Molecular evolutionary genetics analysis (MEGA) software version 4.0. Mol. Biol. Evol. 24:1596-1599.

29. Valenta, V. 1959. Interference studies with yellows-type plant viruses. II. Cross protection tests with European and American viruses. Acta Virol. (Prague) 3:145-152. 